\title{
Continuous and rapid sound regulation via a compact linear electroacoustic field effect transistor
}

\author{
Jingjing Ding, ${ }^{1}$ Xiaole Wang $\odot,{ }^{1,2, *}$ Limin Gu $\odot,{ }^{1}$ Senlei Li $\odot,{ }^{1}$ Xudong Luo, ${ }^{3}$ \\ Chunyu Zhao, ${ }^{1,2}$ and Zhenyu Huang ${ }^{1,2}$ \\ ${ }^{1}$ School of Sensing Science and Engineering, School of Electronic Information and Electrical Engineering, \\ Shanghai Jiao Tong University, Shanghai 200240, China \\ ${ }^{2}$ Institute of Marine Equipment, Shanghai Jiao Tong University, Shanghai 200240, China \\ ${ }^{3}$ School of Physics and Astronomy, Key Laboratory of Artificial Structures and Quantum Control (Ministry of Education), \\ and National Demonstration Center for Experimental Physics Education, Shanghai Jiao Tong University, Shanghai 200240, China
}

(Received 10 March 2021; revised 8 December 2021; accepted 10 December 2021; published 21 December 2021)

\begin{abstract}
We report a simple realization of a compact linear electroacoustic field effect transistor (EA-FET) constructed by coupling a membrane-type acoustic metamaterial with a piezoelectric shunt circuit. As analogous to the electroelectronic field effect transistor, the proposed EA-FET is a four-terminal device in which the sound flux is regulated by the external electric field. The core function of the EA-FET relies on the electrical-elasticitycontrol mechanism stemming from the inside mechanical and electrical interactions. The effectiveness of the EA-FET concept is proved by investigating the intrinsic output and transfer properties and the sound transmission characteristics. The continuous and rapid regulation of sound flux, as well as the acoustic binary encoding via an EA-FET sample, were demonstrated experimentally. As an essential analog acoustic functional device, the EA-FET could play a significant role in engineering the integration of analog acoustic circuits, and it may be adapted to build distributed information processing systems.
\end{abstract}

DOI: 10.1103/PhysRevResearch.3.043206

\section{INTRODUCTION}

The booming development of digital electronic integrated circuits has become one of the primary driving forces of the current technological progress. Although digital electronic integrated circuits can perform various types of general-purpose computations and thus have wide applicability, their development today stands on the brink of a precipice. On the one hand, the manufacturing of silicon-based chips will reach the process limit in the near future, rendering Moore's law somehow irrelevant; on the other hand, the low speed, high power consumption, and the system complexity caused by analog-to-digital conversions in digital electronic integrated circuits, greatly restrict the efficiency of massively parallel operations. Motivated by this fact, there has recently been a surge of interest in performing quantum computations and wave-based analog computations [1-3]. As a more technically mature solution, wave-based analog computations essentially avoid analog-to-digital conversions to allow massively parallel operations [4]. In particular, artificially designed photonic and phononic structures [5-9], i.e., metamaterials, have been proposed to support ultrafast responses, high throughputs, energy conversionless operations, and subwavelength profiles,

\footnotetext{
*Corresponding author: lelemyworld@sjtu.edu.cn

Published by the American Physical Society under the terms of the Creative Commons Attribution 4.0 International license. Further distribution of this work must maintain attribution to the author(s) and the published article's title, journal citation, and DOI.
}

which enable miniaturization and integration of wave-based analog computers unlike their mechanical ancestors [2].

Due to the inherent transport similarity among electrons, photons, and phonons, constructions of analog acoustic integrated circuits have received increasing attention inspired by their electronic and electromagnetic counterparts [10], which may have giant potential applications, such as adaptive noise barrier, high-resolution biomedical imaging, and large-scale acoustic information parallel processing [11-13]. At present, several elementary analog acoustic functional devices, e.g., acoustic diodes [14-18], acoustic transistors [19-23], acoustic modulators [24-29], acoustic switches [30-36], and acoustic demultiplexers [37-41], have been proposed to realize acoustic nonreciprocal transmissions, acoustic logic gates, and acoustic digital encoders in subwavelength scales. Note that, in analog/digital electronic integrated circuits, as a crucial device, the electroelectronic field effect transistor (EE-FET), e.g., the metal oxide semiconductor field-effect transistor (MOSFET), exerts an augmented electric field to control the current flow from the source terminal to the drain terminal. EE-FET can work either in the switching states to realize the logic on/off switching, or in the intermediate states, regarded as a variable resistance or conductance, to realize the continuous regulation of current flow. Therefore, EEFET is widely harnessed for power amplifications, circuit switches, and waveform rectifications. In the current context of the acoustic analog computing paradigm, effective implementation of the acoustic counterpart of EE-FET, i.e., the acoustic field effect transistor, is of paramount importance to promote the development of analog acoustic integrated circuits. 


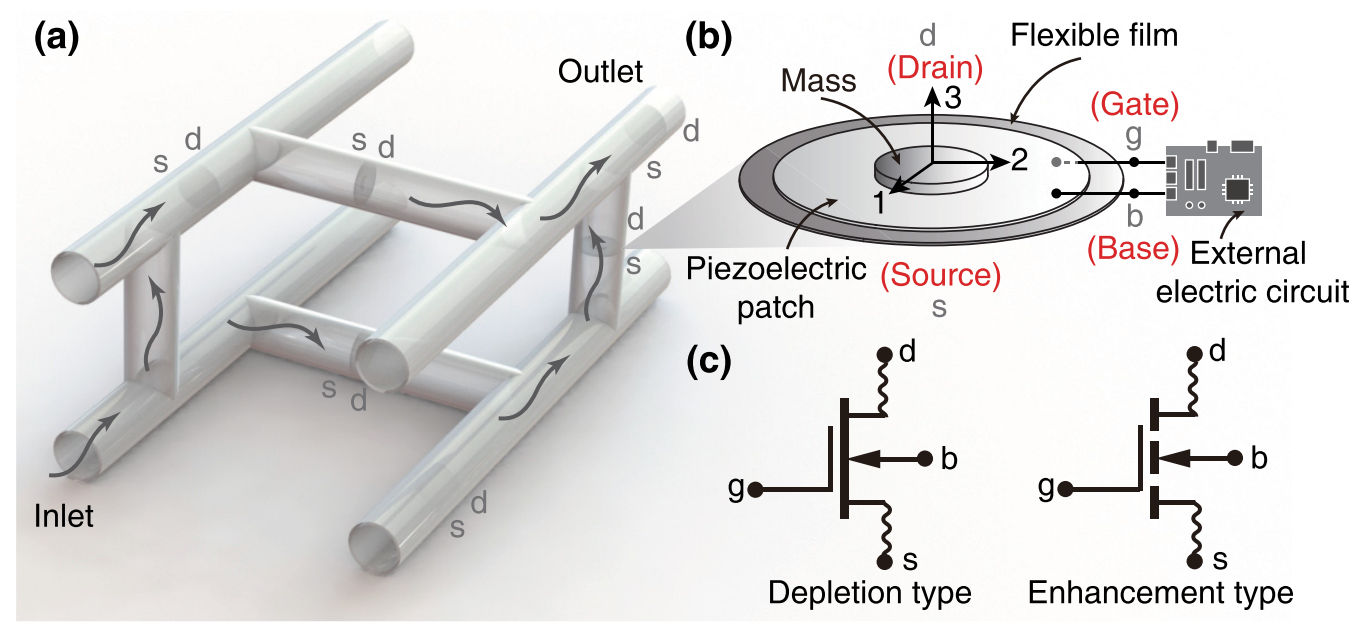

FIG. 1. (a) Schematic drawing of a multidirectional acoustic circuit equipped with some EA-FETs. Arrows indicate directions of the sound flux through each EA-FET from the source terminal (labeled by $s$ ) to the drain terminal (labeled by $d$ ). (b) The detailed constructions of an EA-FET unit cell. Cartesian orthogonal coordinates are assigned to the piezoelectric patch. (c) Equivalent circuit symbols of the depletion-type and the enhancement-type EA-FET imitating EE-FET (e.g., MOSFET).

The strictly acoustic analog of EE-FET is the acoustoacoustic field effect transistor (AA-FET). Conceptually, AA-FET is a device in which the variation in the augmented acoustic field determines the sound flux through the device. Although AA-FET is impeccable from a physical perspective, for analog acoustic integrated circuits built with AA-FETs at the practical application level, the limited speed of sound traveling in materials poses serious implementation challenges: (i) significant delay in responding to the input acoustic waves; (ii) import high energy to drive, requiring high levels of external energy supply; (iii) difficulty in achieving a compact device size, especially when handling low-frequency sound signals of several hundred hertz; and (iv) severe frequency dependence of the operating state, reducing the ability to resist noise interferences. By abandoning the premise that acoustic computing and electronic computing are strictly analogous, we can explore a variety of possibilities. A feasible approach is to control the acoustic field using an augmented electric field, namely an electroacoustic field effect transistor (EAFET). To this end, an electromechanical coupling mechanism with high sensitivities and low delays must be introduced into EA-FET. The utilization of piezoelectric materials with tunable external electric circuits, as has been demonstrated for vibration control [42], energy harvesting [43], adjustable directivity [44], unidirectional devices [13,45], and adaptive elastic metamaterials [46,47], may be a promising candidate. Moreover, to realize the rectification of airborne sound waves through a subwavelength scale device, the membrane-type acoustic metamaterial [48] may suitably be employed as the host structure to couple with the piezoelectric component.

In this paper, we introduce a piezoelectric component with electrically controllable elasticity into a membranetype acoustic metamaterial to build a basic configuration of EA-FET. Despite being a nonstrict acoustic counterpart of EE-FET, the sound flux from the source terminal to the drain terminal of the proposed EA-FET can be regulated continuously and rapidly by the external electric circuit. A comprehensive investigation of the intrinsic output and trans- fer properties and the sound transmission characteristics of EA-FET is performed through theoretical predictions, numerical simulations, and experimental measurements. The acoustic binary encoding via EA-FET is demonstrated experimentally in a one-dimensional acoustic circuit. These results reveal the underlying physics and potential applications of an underexplored class of acoustic field effect transistor.

\section{BASIC CONCEPT OF EA-FET}

As shown in Fig. 1, the proposed EA-FET is a fourterminal device, which consists of a membrane-type acoustic metamaterial with the "source" and "drain" terminals (represented by $s$ and $d$ ) and an embedded piezoelectric shunt circuit, i.e., the piezoelectric patch and its shunted external electric circuit, with the two adjustable electrodes (represented by $g$ and $b$ ). The membrane-type acoustic metamaterial is composed of a flexible film as the substrate, a piezoelectric patch bonded to the flexible film, and a centrally placed mass for adjusting the mechanical resonance frequencies. The main function of the membrane-type acoustic metamaterial is to generate a certain acoustic response controlled by the external electric circuit for quantitatively regulating the sound flux [see Fig. 1(a)]. It is straightforward to tailor the geometric dimensions and constituent materials of the membrane-type acoustic metamaterial to produce desirable EA-FET with appropriate sizes and operating frequencies.

In EA-FET, the piezoelectric patch plays a critical role in exchanging energy between the mechanical domain and the electrical domain. More specifically, when the piezoelectric patch is shunted by an external electric circuit, initial deformations (or strains, due to Hooke's law) of the piezoelectric patch induced by sound wave excitations can generate bound charges on the piezoelectric patch surface due to the direct piezoelectric effect. These bound charges can be easily compensated by free charges transferred from the external electric circuit. Consequently, the current of charges produces a voltage change that is fed back to the piezoelectric patch to cause additional deformations due to the inverse piezoelectric effect. 
Since the induced voltage depends on the internal impedance of the piezoelectric patch and the external impedance of the external electric circuit, the total deformations, i.e., the sum of the initial and the additional deformations, of the piezoelectric patch can be effectively controlled by the piezoelectric shunt circuit, which is referred to as the electrical elasticity control [49]. Hence, the effectiveness of EA-FET strongly depends on the designed piezoelectric shunt circuit.

According to the charge generation principle of piezoelectric materials, opposite charges produced on the same surface of the piezoelectric patch in the vibration modes with out-of-phase motions can counteract the piezoelectric effect. Therefore, to obtain the strongest electrical elasticity control capability, the first-order transverse mode of EA-FET is selected as the operating mode to avoid undesirable charge cancellations. In this mode, the piezoelectric patch and the mass are in a translational motion. Therefore, as shown in Fig. 1(b), only the uniaxial stress applied in the 1-axis direction (and the 2-axis due to the axisymmetry) and the nonzero component of the electric field generated in the 3-axis direction are considered [49]. Moreover, since the piezoelectric patch does not produce local deformations in this mode, it is reasonable to assume a uniform spatial distribution of the electric field and the electric displacement of the piezoelectric patch. Thus, in the frequency domain, the governing constitutive equations for the piezoelectric shunt circuit can be expressed as [50]

$$
\left[\begin{array}{c}
I \\
S_{1}
\end{array}\right]=\left[\begin{array}{cc}
\left(Z_{3}^{\mathrm{E}}\right)^{-1} & j \omega A d_{31} \\
d_{31} / h & s_{1}^{\mathrm{E}}
\end{array}\right]\left[\begin{array}{c}
V \\
T_{1}
\end{array}\right],
$$

where $I$ and $V$ are the externally applied current and voltage, respectively. $S_{1}$ and $T_{1}$ are the elastic strain and stress in the 1 -axis direction, respectively. $s_{1}^{\mathrm{E}}$ is the elastic compliance at a constant electric field. $d_{31}=k_{31} \sqrt{s_{1}^{\mathrm{E}} \varepsilon_{3}^{\mathrm{T}}}$ is the charge constant for the transverse case, i.e., the mechanical force is applied in the 1-axis direction, while the electric field is generated in the 3-axis direction. $k_{31}$ is the electromechanical coupling coefficient, and $\varepsilon_{3}^{\mathrm{T}}$ is the dielectric constant under constant elastic stress. $A$ and $h$ are the area and thickness of the piezoelectric patch, respectively. $\omega$ is the angular frequency, and $j=\sqrt{-1}$ is the imaginary unit. Since the external electric circuit is in parallel connected to the piezoelectric patch, the electrical impedance $Z_{3}^{\mathrm{E}}$ of the shunted piezoelectric patch can be expressed as

$$
\left(Z_{3}^{\mathrm{E}}\right)^{-1}=\left(Z_{3}^{\mathrm{ED}}\right)^{-1}+\left(Z_{3}^{\mathrm{EL}}\right)^{-1},
$$

where $Z_{3}^{\mathrm{ED}}=1 /\left(j \omega C_{\mathrm{pT}}\right)$ and $Z_{3}^{\mathrm{EL}}=1 /\left(j \omega C^{\mathrm{EL}}\right)$ are the internal and external impedances, respectively. $C_{\mathrm{pT}}=A \varepsilon_{3}^{\mathrm{T}} / h$ and $C^{\mathrm{EL}}$ are the equivalent capacitances of the piezoelectric patch and the external electric circuit, respectively. As with EE-FET, EA-FET draws no current from the external electric circuit, so we have $I=0$. Thus, Eq. (2) can be substituted into Eq. (1) to obtain an expression for the mechanical impedance $Z_{3}^{\mathrm{M}}$ of the shunted piezoelectric patch,

$$
Z_{3}^{\mathrm{M}}=\frac{T_{1}}{S_{1}}=\frac{(j \omega)^{-1} K_{\mathrm{oc}}\left(1-k_{31}^{2}\right)}{1-k_{31}^{2}\left[C_{\mathrm{pT}} /\left(C_{\mathrm{pT}}+C^{\mathrm{EL}}\right)\right]},
$$

(a)
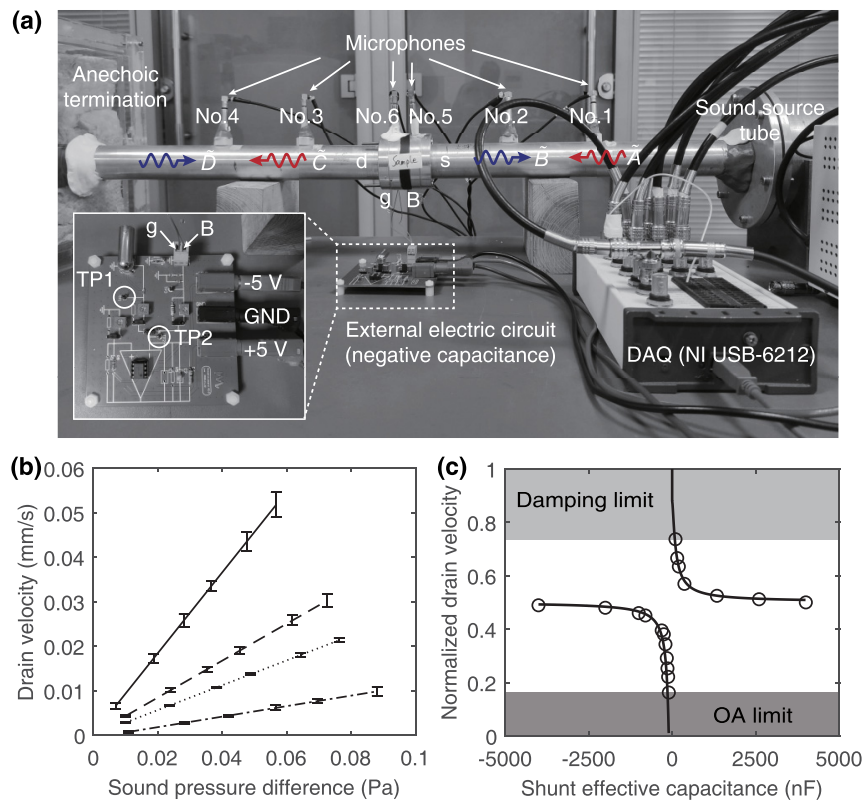

FIG. 2. (a) Experimental setup of the EA-FET sample inserted in a one-dimensional sound tube. (b) Intrinsic output properties, i.e., the sound pressure difference between the $s$ and $d$ terminals vs the drain velocity. The experimental results correspond to four configurations, i.e., open circuit (solid line), shunt effective capacitance $C^{\mathrm{EL}}=-200 \mathrm{nF}$ (dashed line), $-120 \mathrm{nF}$ (dotted line), and $-80 \mathrm{nF}$ (dash-dotted line). Error bars present the standard deviations resulting from the least-squares fit over three EA-FET samples. (c) Intrinsic transfer properties, i.e., the normalized drain velocity vs $C^{\mathrm{EL}}$. Experimental and simulated results are represented by circles and curves, respectively. The drain velocities are normalized to the drain velocity in the case of total transmission, i.e., without EA-FET.

where $K_{\mathrm{oc}}$ is the stiffness of the piezoelectric patch with the open circuit $\left(C^{\mathrm{EL}}=0\right)$. Equation (3) describes the quantitative relationship between $C^{\mathrm{EL}}$ and $Z_{3}^{\mathrm{M}}$. By adjusting $C^{\mathrm{EL}}$, we can alter $Z_{3}^{\mathrm{M}}$ accordingly to control the sound flux through EA-FET.

\section{INTRINSIC OUTPUT AND TRANSFER PROPERTIES}

Elucidation of the intrinsic output and transfer properties of the proposed EA-FET is fundamental for future designs and applications. Therefore, an EA-FET sample was fabricated and inserted into a one-dimensional sound tube to measure the variations of sound pressures and particle velocities between the $s$ and $d$ terminals with the shunt effective capacitance $C^{\mathrm{EL}}$ between the $g$ and $b$ terminals.

\section{A. EA-FET sample and experimental setup}

As shown in Fig. 2(a), all the electronic components involved in the external electric circuit were assembled on a surface-mount circuit board. Refer to Appendix A for details about the EA-FET sample. In experiments, sound waves with a tone frequency of $910 \mathrm{~Hz}$ generated from a loudspeaker installed in the sound source tube impinge on and transmit through the EA-FET sample, and finally are almost completely absorbed by the anechoic termination (porous 
materials with 1-m thickness). Along the sound tube, six pressure-field microphones (BSWA, type MPA416) were employed to probe the sound pressure signals at the far-field $(>100 \mathrm{~mm})$ and near-field $(\sim 10 \mathrm{~mm})$ positions away from the EA-FET sample surface. A data acquisition (DAQ) device (NI, type USB-6212) was used to record the six sound pressure signals, which were then postprocessed in a customized MATLAB program. More specifically, the sound pressure signals $\left(P_{5}\right.$ and $\left.P_{6}\right)$ detected at the two near-field positions (no. 5 and no. 6 microphones) directly represent the upstream and downstream sound pressures across the EA-FET sample, which can be further used to calculate the sound pressure difference $\left(\triangle P=P_{5}-P_{6}\right)$ and the drain velocity $\left(V_{\mathrm{D}}=P_{6} / Z_{0}\right.$, where $Z_{0}$ is the characteristic impedance of air); based upon the transfer-matrix approach [51], the sound pressure signals $\left(P_{1}-P_{4}\right)$ probed at the four far-field positions (no. 1-no. 4 microphones) are adopted to decompose the positive-going (with amplitudes of $\tilde{A}$ and $\tilde{C}$ ) and negative-going (with amplitudes of $\tilde{B}$ and $\tilde{D}$ ) plane-wave components propagating in the upstream and downstream tube segments. For the case of an anechoic termination used in our experiments where $\tilde{D}$ vanishes, the measured acoustic transmittance $\tau$ and acoustic reflectance $\gamma$ of the EA-FET sample can be obtained by $\tau=|\tilde{C} / \tilde{A}|^{2}$ and $\gamma=|\tilde{B} / \tilde{A}|^{2}$, respectively.

\section{B. Intrinsic output properties}

Figure 2(b) shows the intrinsic output properties of the drain velocity as a function of the sound pressure difference between the $s$ and $d$ terminals of the EA-FET sample operated under four typical configurations of the external electric circuit. It can be found that, under each configuration, the drain velocity and the sound pressure difference show an obvious linear relationship, which indicates that the proposed EA-FET works in the linear regime. Moreover, the slope of each line, i.e., the reciprocal of acoustic impedance, increases as the provided $C^{\mathrm{EL}}$. With this in mind, EA-FET could be treated as a linear electroacoustic device that is dominated by electrically adjustable transverse mechanical resonances.

For our EA-FET sample, the frequency of the first-order transverse mode is designed to be $910 \mathrm{~Hz}$ exactly matching the incident frequency of sound waves. Under the condition of a deep subwavelength scale, i.e., the entire thickness of EA-FET is about $1 / 313$ wavelength at $910 \mathrm{~Hz}$, the mechanical deformations of EA-FET are much smaller than the wavelengths of incident sound waves, and hence the flexible film, the piezoelectric patch, and the mass are subjected to a uniform force action of the sound pressure difference $\Delta P$. EA-FET operating in the first-order transverse mode can thus be regarded as a single-degree-of-freedom system with lumped mass and stiffness parameters.

The term "acoustic impedance $\left(Z^{\mathrm{A}}\right)$ " is adopted here to fully describe the dynamic characteristics of EA-FET and its interactions with the adjacent air domain. According to Newton's second law, $Z^{\mathrm{A}}$ can be expressed as

$$
Z^{\mathrm{A}}=\varphi \frac{\Delta P}{j \omega X_{3}}=\varphi\left[j \omega M+\frac{K_{\mathrm{N}}+K_{\mathrm{T}}}{j \omega}(1+j \eta)\right],
$$

where $X_{3}$ is the averaged displacement in the 3 -axis direction. $M, K_{\mathrm{N}}$, and $K_{\mathrm{T}}=j \omega Z_{3}^{\mathrm{M}}$ are the first-order modal mass, nontunable stiffness, and tunable stiffness, respectively. $\varphi$ with a dimension of $1 / \mathrm{m}^{2}$ is a coefficient associated with the mode shape. The loss factor $\eta$ is introduced to assess all the energy dissipation caused by the damping effect.

Following Eq. (3), for the region in which $C^{\mathrm{EL}}$ is positive, the tunable stiffness $K_{\mathrm{T}}$ can only be varied from $K_{\mathrm{oc}}\left(1-k_{31}^{2}\right)$ with the short circuit $\left(C^{\mathrm{EL}}=\infty\right.$, or $\left.C_{\mathrm{pT}}=0\right)$ to $K_{\mathrm{oc}}$ with the open circuit. However, for the region where $C^{\mathrm{EL}}$ is negative, $K_{\mathrm{T}}$ can be theoretically tuned from $0\left(C^{\mathrm{EL}}=-C_{\mathrm{pT}}\right)$ to $\infty$ $\left[C^{\mathrm{EL}}=-C_{\mathrm{pT}}\left(1-k_{31}^{2}\right)\right]$. Therefore, the use of negative $C^{\mathrm{EL}}$ can significantly increase the adjustment range of the total stiffness $\left(K_{\mathrm{N}}+K_{\mathrm{T}}\right)$.

Note that the piezoelectric shunt circuit with a negative capacitance is essentially a feedback control system, and hence the condition of stable operations needs to be satisfied first. Since the negative capacitance shunt circuit only modulates the electronic circuit without any mechanical modifications, the stability of the entire system depends on the stability of the electronic circuit system. In terms of the negative capacitance shunt circuit employed in the EA-FET sample, $\left|C^{\mathrm{EL}}\right|>\left|C_{\mathrm{pT}}\right|$ is required for stable operations to ensure that the feedback loop gain for the operational amplifier (OA) must be negative [44]. Thus, from Eq. (3), $C^{\mathrm{EL}}$ altered from $-C_{\mathrm{pT}}$ to $-\infty$ can lead to an adjustment of $K_{\mathrm{T}}$ from 0 to $K_{\mathrm{oc}}\left(1-k_{31}^{2}\right)$, which implies that a softening mechanism is introduced into EA-FET compared with that of the open-circuit configuration. In addition, according to Eqs. (3) and (4), if a resonance state of EA-FET with the open circuit occurs, i.e., $\omega=\sqrt{\left(K_{\mathrm{N}}+K_{\mathrm{T}}\right)(1+j \eta) / M}, Z^{\mathrm{A}}$ would reach its minimum value, which confirms that EA-FET is in the state where the upper bound of the drain velocity could be achieved, as shown in Fig. 2(b).

\section{Intrinsic transfer properties}

For the fabricated EA-FET sample with $C^{\mathrm{EL}}$ altered from $-C_{\mathrm{pT}}$ to $-\infty$, the drain velocity can be regulated accordingly, which can be completely demonstrated in the intrinsic transfer properties, as shown in Fig. 2(c). To fully present the intrinsic transfer properties of EA-FET, besides the experimental results corresponding to the negative $C^{\mathrm{EL}}$, Fig. 2(c) also shows the experimental results corresponding to the positive $C^{\mathrm{EL}}$, but those results were obtained by employing several discrete positive capacitances. Moreover, the simulated results based upon a finite-element model (see Appendix B) conducted in COMSOL MULTIPHYSICS are also shown for comparison. Good agreement between experimental measurements and numerical simulations can be observed, except for the two highlighted areas.

The complete intrinsic transfer properties of EA-FET exhibit a hyperbolic feature, in which the left and right branches separated by the open-circuit configuration correspond to the negative and positive capacitance regimes, respectively. For the negative capacitance regime, due to the stability condition of the employed OA, the minimum achievable value of $C^{\mathrm{EL}}$ is $-C_{\mathrm{pT}}$, that is, $-78 \mathrm{nF}$ for the adopted piezoelectric patch. In comparison to the simulated results, the experimental results cannot reach such a small value of the normalized drain 
velocity, i.e., the OA limit. With the increase of negative capacitances, the normalized drain velocity tends to be that under the short-circuit configuration, where $K_{\mathrm{T}}$ is equal to $K_{\mathrm{oc}}\left(1-k_{31}^{2}\right)$. For the positive capacitance regime, the adjustment range of $K_{\mathrm{T}}$ is barely from $K_{\mathrm{oc}}$ to $K_{\mathrm{oc}}\left(1-k_{31}^{2}\right)$. The drain velocity can reach the maximum value in the resonant state of EA-FET with the open circuit. However, the entire system contains a certain amount of mechanical and electrical damping, reducing the resonant response amplitude of the EA-FET sample (see Appendix B). Therefore, compared with the simulated results, the experimental results cannot hit such a large value of the normalized drain velocity, i.e., the damping limit. As the positive capacitance increases, the normalized drain velocity also approaches that of the short-circuit configuration.

The intrinsic transfer properties shown in Fig. 2(c) confirm that using the piezoelectric shunt circuit can regulate the sound flux, i.e., the normalized drain velocity, between 0 and 1 continuously. The shunted negative capacitance supports a wider adjustment range for the sound flux than the shunted positive capacitance. Especially in switching between 0 and 1 states of EA-FET, we can only rely on the negative capacitance shunt circuit to change between $C^{\mathrm{EL}} \sim-C_{\mathrm{pT}}$ and the open circuit, respectively. Moreover, of special interest is the remarkable continuous dynamic adjustment range that occurs when $C^{\mathrm{EL}}$ is close to $-C_{\mathrm{pT}}$, which benefits the efficient regulation of the intermediate states of EA-FET.

Although the intrinsic output and transfer properties shown in Figs. 2(b) and 2(c) are for the EA-FET sample with specific geometric and material parameters, such intrinsic properties are in common for our EA-FET configuration. Considering the trend toward increasing integration of analog acoustic computing, we further fabricated a scaled-down EA-FET sample to demonstrate the universality and scalability of our EA-FET configuration. See Appendix C for the experimental results on its intrinsic output and transfer properties.

The kind of EA-FET studied in Figs. 2(b) and 2(c) is exactly designed to be in a resonant state with the open circuit, which ensures that this kind of EA-FET is a normally on device, i.e., the maximum sound flux is obtained under the open-circuit configuration. Once the external electric circuit is shunted, the acoustic impedance of EA-FET can be increased, thereby reducing the acoustic flux. Hence, to some extent, this kind of EA-FET can be analogous to the depletion-type MOSFET, in which a channel is already constructed physically and the applied voltage would narrow the channel width to attenuate the conductivity. We refer to this kind of EA-FET as the depletion-type EA-FET. Interestingly enough, as a wave-based device, EA-FET may behave as different types under different excitation frequencies. Due to the softening mechanism introduced into EA-FET through the external electric circuit, the same EA-FET sample can also be analogous to the enhancement-type MOSFET by adjusting the incident frequency from $910 \mathrm{~Hz}$ to another lower value, e.g., $820 \mathrm{~Hz}$. See Appendix C for the intrinsic output and transfer properties of the same EA-FET sample operating at 820 Hz. In analogy to MOSFET, Fig. 1(c) shows the equivalent circuit symbols for the depletion-type and enhancement-type EA-FET.

\section{SOUND TRANSMISSION CHARACTERISTICS}

Because of the resonant nature, the frequency-dependence issue is inevitable for EA-FET. The proposed analytical model also allows us to estimate the sound transmission characteristics of EA-FET in the frequency domain, which may provide insights into the frequency-dependence issue of this kind of metamaterial-based functional device. Since EA-FET is of a deep subwavelength profile, the one-dimensional sound transmission characteristics of EA-FET operating in a certain state can be described by the transfer-matrix equation,

$$
\left[\begin{array}{l}
p^{-} \\
v^{-}
\end{array}\right]=\left[\begin{array}{cc}
1 & Z^{\mathrm{A}} \\
0 & 1
\end{array}\right]\left[\begin{array}{l}
p^{+} \\
v^{+}
\end{array}\right]
$$

where $p^{-}, v^{-}$and $p^{+}, v^{+}$denote the sound pressures and particle velocities at the front and back surfaces of EA-FET. Omitting the time dependence $e^{j \omega t}$ for simplicity, we have

$$
\begin{aligned}
& p^{-}=\tilde{A}+\tilde{B}, \quad v^{-}=(\tilde{A}-\tilde{B}) / Z_{0}, \\
& p^{+}=\tilde{C} e^{-j k_{0} d}, \quad v^{+}=\tilde{C} e^{-j k_{0} d} / Z_{0},
\end{aligned}
$$

where $k_{0}$ is the wave number in air, and $d$ is the EA-FET thickness. The acoustic transmittance $\tau$ and the acoustic reflectance $\gamma$ can be derived by substituting Eq. (6) into Eq. (5),

$$
\begin{aligned}
& \tau=\left|\frac{\tilde{C}}{\tilde{A}}\right|^{2}=\left|\frac{2 Z_{0}}{2 Z_{0}+Z^{\mathrm{A}}}\right|^{2}, \\
& \gamma=\left|\frac{\tilde{B}}{\tilde{A}}\right|^{2}=\left|\frac{Z^{\mathrm{A}}}{2 Z_{0}+Z^{\mathrm{A}}}\right|^{2} .
\end{aligned}
$$

By solving Eqs. (7) and (8) together, we get

$$
Z^{\mathrm{A}}=\psi Z_{0} \pm j Z_{0} \sqrt{\frac{4}{\hat{\tau}}-\psi^{2}-4 \psi-4},
$$

where $\psi=(1-\gamma) / \tau-1$. In terms of an ideal EA-FET without any energy dissipation $(\eta=0)$, there is a deterministic relationship between $\tau$ and $\gamma$, that is, $\gamma=1-\tau$. Thus, according to Eq. (9), it can attain two bound states, i.e., the total transmission $(\tau=1)$ and the total reflection $(\tau=0)$, corresponding to the acoustic impedance of $Z^{\mathrm{A}}=0$ and $Z^{\mathrm{A}}=$ $\infty$, respectively. Between the two bound states, the acoustic impedance $Z^{\mathrm{A}}$ can be modified to achieve the continuous adjustment of $\tau$ from 1 to 0 . Substitution of Eq. (4) with $\eta=0$ into Eq. (9) yields

$$
\tau=\frac{4 Z_{0}^{2}}{4 Z_{0}^{2}+\varphi^{2}\left(\omega M-\frac{K_{\mathrm{N}}}{\omega}-\frac{K_{\mathrm{oc}}}{\omega} \frac{1-k_{21}^{2}}{1-\frac{k_{31}^{2} \mathrm{c}_{\mathrm{PT}} \mathrm{TL}}{C_{\mathrm{p} \mathrm{T}} \mathrm{CL}}}\right)^{2}} .
$$

Equation (10) establishes the explicit relationship between the target $\tau$ and the required $C^{\mathrm{EL}}$ for an ideal EA-FET. The simulated $\tau$ of our EA-FET sample as a function of both frequencies and $C^{\mathrm{EL}}$ is illustrated in Fig. 3. We can find that the peak values of $\tau$ mainly concentrated between 840 and $940 \mathrm{~Hz}$ present an obvious frequency-dependence distribution feature. Nevertheless, when $C^{\mathrm{EL}}$ is close to $-C_{\mathrm{pT}}$, a relatively broadband high-value $\tau$ could be attained, indicating that the acoustic impedances of EA-FET are tuned to match well with the characteristic impedance of air in a broadband frequency range by shunting such negative capacitances. As predicted 


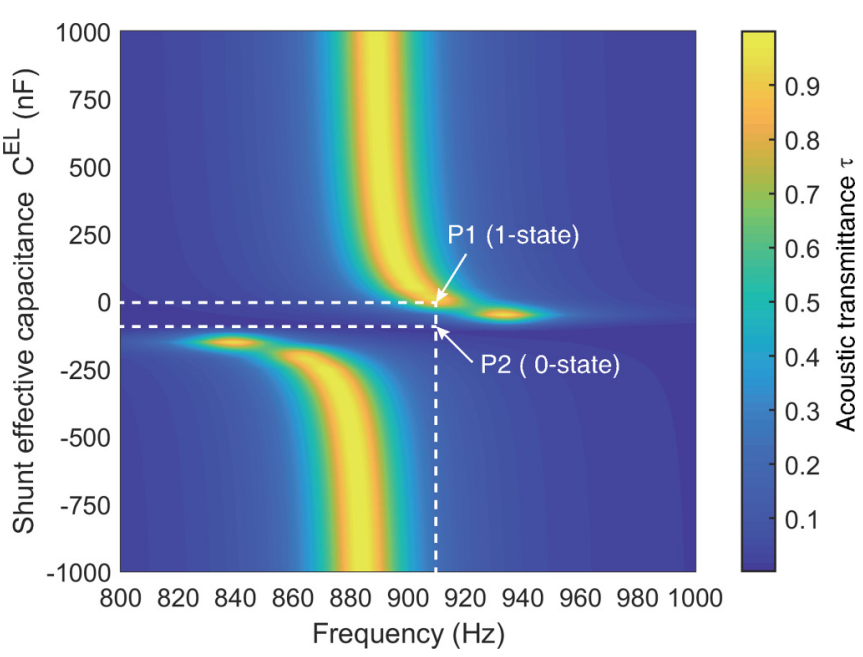

FIG. 3. Simulated acoustic transmittance $\tau$ of the EA-FET sample varying with frequencies and shunt effective capacitances $C^{\mathrm{EL}}$. To completely present the frequency dependence of EA-FET, $C^{\mathrm{EL}}$ still gives positive and negative values. The corresponding $C^{\mathrm{EL}}$ for $\mathrm{P} 1$ and $\mathrm{P} 2$ are $0 \mathrm{nF}$, i.e., open circuit, and $-C_{\mathrm{pT}}$, i.e., $-78 \mathrm{nF}$, respectively.

from Eq. (10), two points denoted as P1 and P2 both located at $910 \mathrm{~Hz}$ are marked in Fig. 3. At P1, EA-FET is with the open circuit, while at P2, EA-FET is shunted by the negative capacitance $C^{\mathrm{EL}} \sim-C_{\mathrm{pT}}$. If the piezoelectric shunt circuit in EA-FET switches from P1 to P2, $\tau$ could be switched from 1 -state to 0 -state correspondingly.

Based upon the transmission-line theory [52], the scattering matrix $\mathbf{S}$ of EA-FET can be straightforwardly calculated from the transfer matrix as

$$
\mathbf{S}=\left[\begin{array}{ll}
S_{11} & S_{12} \\
S_{21} & S_{22}
\end{array}\right]=\left[\begin{array}{cc}
\frac{Z^{\mathrm{A}}}{2 Z_{0}+Z^{\mathrm{A}}} & \frac{2 Z_{0}}{2 Z_{0}+Z^{\mathrm{A}}} \\
\frac{2 Z_{0}}{2 Z_{0}+Z^{\mathrm{A}}} & \frac{Z^{\mathrm{A}}}{2 Z_{0}+Z^{\mathrm{A}}}
\end{array}\right] .
$$

Such a scattering matrix fulfills the transmission symmetry and reflection symmetry, which implies that the source and drain terminals of EA-FET can be used interchangeably with no change in the intrinsic output and transfer properties. This interchangeability is also held by some types of EE-FET. The scattering matrix in Eq. (11) applies to EA-FET operating in a certain state, and thus it obeys the reciprocity, i.e., $S_{21}=S_{12}$. However, EA-FET possessing time-modulated properties can also be remolded as a time-variant system that may break time-reversal symmetry $[13,53]$, especially for the EA-FET cascaded cases, to bring some inspirations to engineer compact acoustic nonreciprocal devices.

\section{DEMONSTRATION OF ACOUSTIC BINARY ENCODING IN REAL TIME}

In the paradigm of electronic computing, information is digitally encoded in the high and low voltage or the strong and weak current, and therefore the acoustic binary encoding through EA-FET is reasonable by analogy, in which information can be encoded effectively in the high and low sound pressure or the strong and weak drain velocity. We experimentally demonstrated the real-time acoustic binary encoding by repeatedly switching the EA-FET sample between P1 (1state) and P2 (0-state) marked in Fig. 3. See the Supplemental Material (Ref. [54]) for a video that showcases a primary pro-
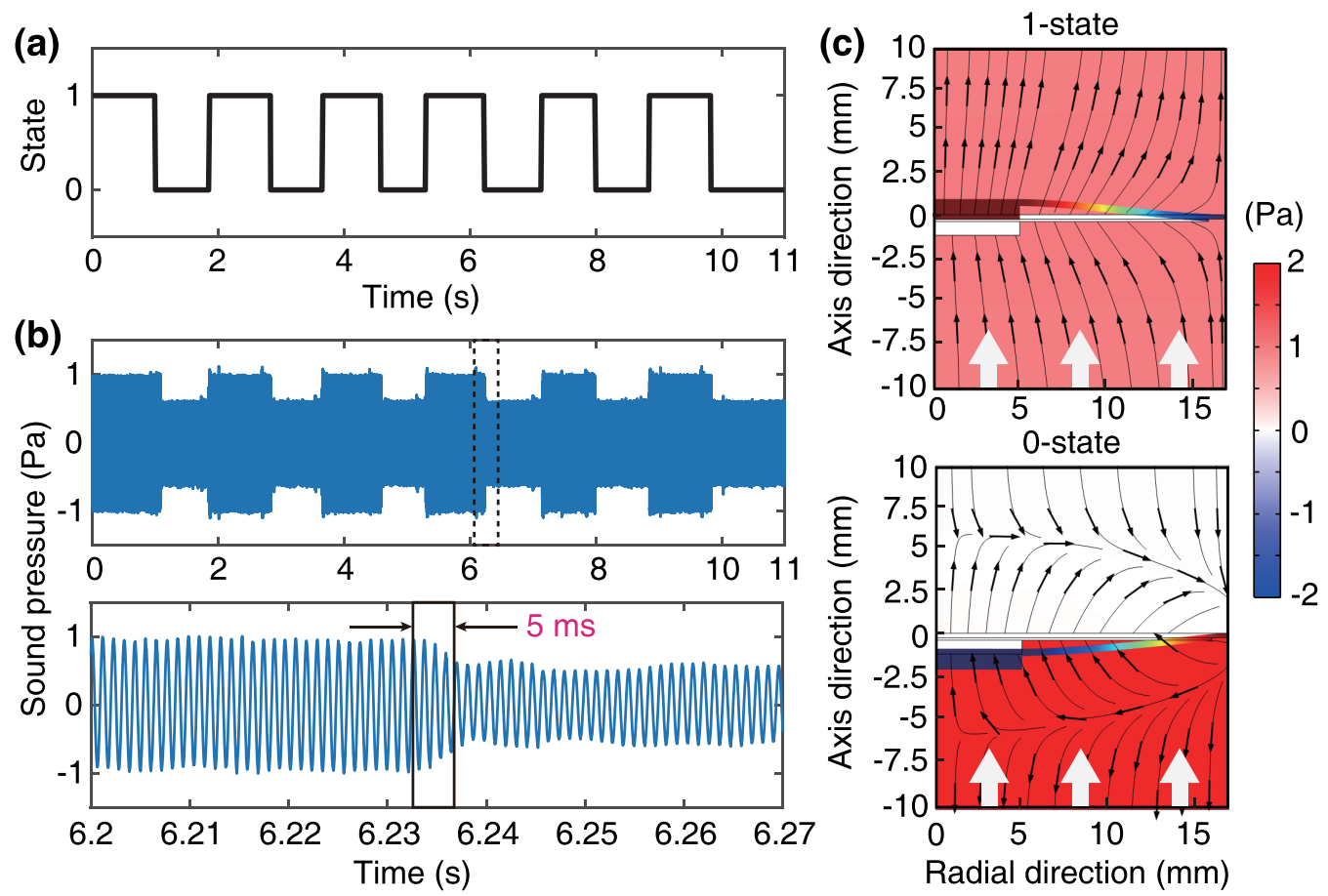

FIG. 4. (a) Conceptual visualization of the two states switching in real time. (b) Time-domain sequence of the sound pressure measured near the $d$ terminal. (c) Simulated distributions of sound pressure fields (color map) in the adjacent air domains of EA-FET in 1- and 0-states. Plane waves (white arrows) with an amplitude of $1 \mathrm{~Pa}$ incident on EA-FET from the bottom at $910 \mathrm{~Hz}$. Local velocity streams are represented by black lines with arrows. 
cess of the acoustic binary encoding by manually switching the two states of EA-FET.

As shown in Fig. 4(a), the ideal 1-state and 0-state correspond to the acoustically transparent state and acoustically opaque state, respectively. In this implementation, the shunt effective capacitance $C^{\mathrm{EL}}$ is set as $-80 \mathrm{nF}$, which is close to $-C_{\mathrm{pT}}$ to fully consider the stable operation of our EA-FET sample. Figure 4(b) shows the sound pressure in the time domain measured by the no. 6 microphone placed near the $d$ terminal. It can be found that, when the EA-FET sample is switched from 1-state to 0-state, the sound pressure amplitudes can be switched from 1 to $0.4 \mathrm{~Pa}$, corresponding to a sound energy jump range of about $8 \mathrm{~dB}$. Furthermore, due to the almost instantaneous response of the piezoelectric shunt circuit, the acoustic binary encoding could be operated in real time with a slight delay of $5 \mathrm{~ms}$ [see the enlarged subplot of Fig. 4(b)].

The simulated distributions of sound pressure fields and local velocity streams corresponding to the two states are illustrated in Fig. 4(c). In 1-state, the sound pressure distributions in the air domain before and after EA-FET are almost the same, indicating that EA-FET is transparent to the incident sound waves and cannot play any obstructing role. As a result, EA-FET does not affect the directions of local velocity streams. In contrast, in 0-state, plenty of sound waves are blocked by EA-FET at the front side, and almost no sound waves are propagating behind it, which indicates that EA-FET is opaque to the incident sound waves and acts as an acoustically hard wall. Compared with 1-state, the local velocity streams around EA-FET exhibit a distinct reverse feature in 0 -state, suppressing the transmission of sound energy into the far-field.

\section{CONCLUSIONS AND REMARKS}

In summary, the feasibility of constructing EA-FET to accurately control the sound flux is presented in analogy to what EE-FET does for the electric current. The easy-to-fabricate EA-FET is created via a membrane-type acoustic metamaterial with a piezoinstrumented microstructure shunted by an external electric circuit. By analyzing the intrinsic output properties, the intrinsic transfer properties, and the sound transmission characteristics, we have revealed the underlying physics of EA-FET. The continuous and rapid transmission regulation and the on-off switch of sound waves through EAFET were realized in a one-dimensional acoustic circuit; in addition, a real-time acoustic binary encoding with 1 - and 0 -states was experimentally demonstrated.

This metamaterial-based EA-FET in a subwavelength scale would be readily served as a versatile tool for enhancing the performance of analog acoustic computing, which may pave the way for the realization of further integrated large-scale acoustic circuits. Subsequent work may consider cascading multiple EA-FETs to build logic gates, arranging an EA-FET array to execute parallel analog computing, etc. Just as its electronic counterpart, EA-FET could have a wide range of industrial impacts.

\section{ACKNOWLEDGMENTS}

This work was supported by the National Natural Science Foundation of China (Grant No. 52003155).

\section{APPENDIX A: SAMPLE FABRICATIONS}

In the EA-FET sample, a circular polycarbonate film with a $0.254-\mathrm{mm}$ thickness and a $22-\mathrm{mm}$ radius was adopted as the (a)

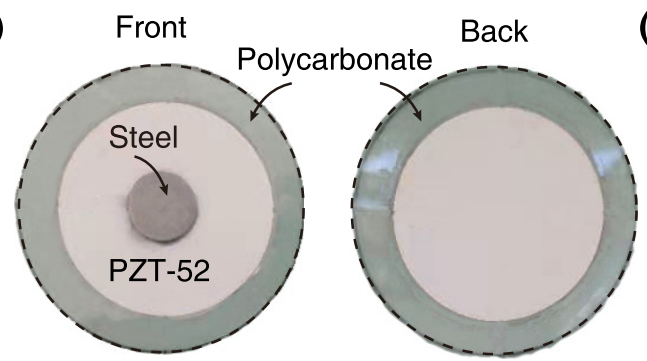

(c)

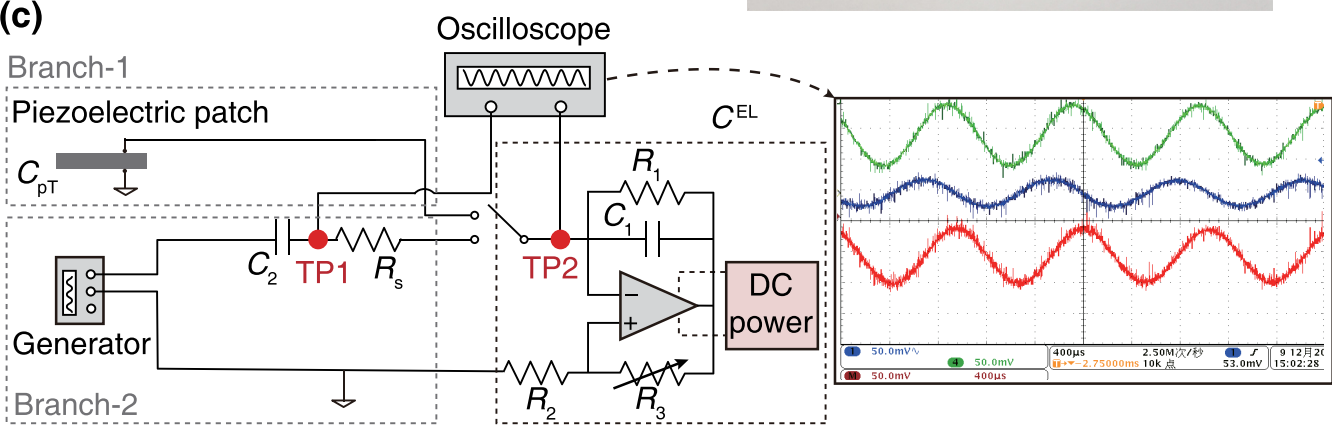

FIG. 5. (a) Photograph of a fabricated EA-FET sample (only the mechanical domain is shown). (b) The EA-FET sample was assembled with a clamped holder. (c) Block diagram of the detailed negative capacitance shunt circuit used in the EA-FET sample. The green and blue curves represent the voltage signals observed from the oscilloscope at Test Point 1 (TP1) and Test Point 2 (TP2), respectively, and the red curve represents the voltage difference between TP2 and TP1. 
substrate. A circular piezoelectric ceramic made of PZT-52 with a $0.15-\mathrm{mm}$ thickness and a $16-\mathrm{mm}$ radius was cut to be the piezoelectric patch. The mass with a radius of $5 \mathrm{~mm}$ was formed by stamping a 0.8-mm-thick stainless steel (type 304) plate. A fast-drying glue (Pattex, type PSK12C-3) was used for the piezoelectric patch to be pasted on the substrate and for the mass to be bonded to the piezoelectric patch center. The photograph of a fabricated EA-FET sample is shown in Fig. 5(a). All lateral edges of the EA-FET sample were fixed by two ring-shaped aluminum holders to form the clamped boundary condition, as shown in Fig. 5(b). Each holder has an inside radius of $17 \mathrm{~mm}$, which matches the inside radius of the sound tube used in experiments.

The material properties of the glue-stiffened polycarbonate film are mass density $\rho=1200 \mathrm{~kg} / \mathrm{m}^{3}$, Young's modulus $E=4 \mathrm{GPa}$, and Poisson's ratio $v=0.37$. The material properties of the PZT-52 piezoelectric patch are mass density $\rho=7600 \mathrm{~kg} / \mathrm{m}^{3}$, Young's modulus $E=56 \mathrm{GPa}$, Poisson's ratio $v=0.36$, elastic compliance $s_{1}^{E}=1.7 \times 10^{-11} \mathrm{~Pa}^{-1}$, dielectric constant $\varepsilon_{3}^{T}=1.45 \times 10^{-8} \mathrm{~F} / \mathrm{m}$, and charge constant $d_{31}=-204 \times 10^{-12} \mathrm{C} / \mathrm{N}, d_{33}=520 \times 10^{-12} \mathrm{C} / \mathrm{N}$. The material properties of the stainless-steel mass are the mass density $\rho=7850 \mathrm{~kg} / \mathrm{m}^{3}$, Young's modulus $E=200 \mathrm{GPa}$, and Poisson's ratio $v=0.3$. The equivalent capacitance of the piezoelectric patch can be calculated by $C_{\mathrm{pT}}=A \varepsilon_{3}^{\mathrm{T}} / h=$ $-78 \mathrm{nF}$.

Figure 5(c) shows the detailed circuit diagram of the negative capacitance shunt circuit exhibited in Fig. 2(a). This circuit board contains two branches, i.e., Branch- 1 and Branch-2. Branch-1 is used for the functional realization of EA-FET. Branch-2 needs to be driven by a signal generator for the independent test of the negative capacitance characteristics. An oscilloscope is adopted to connect TP1 and TP2 to directly observe the voltage and current signals. The voltage measured at TP2 represents the input voltage of the negative capacitance shunt circuit, and the input current of the negative capacitance shunt circuit can be represented by the voltage difference between TP2 and TP1 divided by the resistance $R_{\mathrm{s}}$. It can be found that the phase of the input voltage leads the input current by $90^{\circ}$, which confirms the effectiveness of the negative capacitance shunt circuit.

The values of the involved electronic components are $R_{1}=$ $44.3 \mathrm{k} \Omega, R_{2}=1 \mathrm{k} \Omega, R_{\mathrm{s}}=1000 \Omega, C_{1}=C_{2}=42.4 \mathrm{nF}$. By changing the resistance $R_{3}$ from 1 to $100 \mathrm{k} \Omega$, we can adjust the shunt effective negative capacitance $C^{\mathrm{EL}}$ continuously. A direct current source (DC Power) provides a stable power supply of $\pm 5 \mathrm{~V}$ for the operational amplifier (LM358AP, TI) in this negative capacitance shunt circuit. The value of $C^{\mathrm{EL}}$ can be calculated by $C^{\mathrm{EL}}=-\left(R_{3} / R_{2}\right) C_{1}$. According to the parameters of the selected electrical components, the adjustment range of $C^{\mathrm{EL}}$ that we can achieve in experiments is from $-42.4 \mathrm{nF}$ to infinity.

\section{APPENDIX B: NUMERICAL SIMULATIONS}

The numerical simulations based upon the finite-element method were performed using the solid mechanics and pressure acoustic modules coupled with the electrostatics and electrical circuit interfaces in COMSOL MULTIPHYSICS 5.6. As shown in Fig. 6(a), this finite-element model consists of
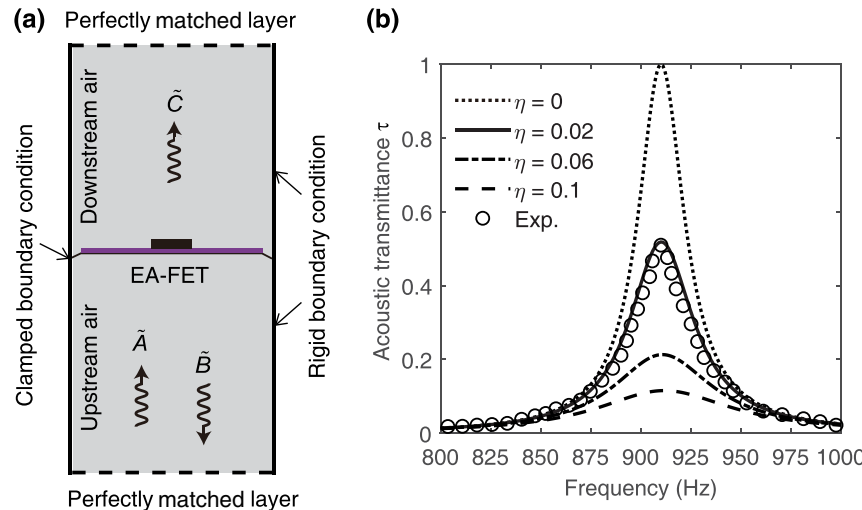

FIG. 6. (a) Schematic drawing of the numerical model of EAFET in a one-dimensional acoustic circuit. (b) Damping effects on the acoustic transmittance $\tau$ of EA-FET with the open circuit.

the EA-FET domain sandwiched between two air domains, i.e., upstream and downstream air domains. Two perfectly matched layers are applied at each end of the air domains to avoid multiple reflections of sound waves. To mimic the experimental conditions, the lateral edges of the flexible film in the EA-FET domain and the two air domains are set to the clamped boundary condition and the rigid boundary condition, respectively. As an acoustic excitation perpendicular to the EA-FET domain, a plane-wave background pressure field with an amplitude of $1 \mathrm{~Pa}$ (i.e., $\tilde{A}=1 \mathrm{~Pa}$ ) is imposed from the upstream air domain. After the interaction with EA-FET, reflected and transmitted waves with amplitudes of $\tilde{B}$ and $\tilde{C}$ are formed in the upstream and downstream air domains, respectively. Two numerical analyses were conducted: the modal analysis of EA-FET with the open circuit to obtain the first-order transverse resonant frequency, and the fully coupled frequency domain analysis to obtain the drain velocities, the acoustic transmittance $\tau$, and the distributions of sound pressure fields in the adjacent air domains of EA-FET in 1and 0 -states.

In actual applications, damping is inevitable due to constituent materials, circuit losses, and production processes, and thus damping becomes one of the important factors affecting the functions of EA-FET. Damping effects can be considered roughly in our numerical simulations by introducing an imaginary part into Young's modulus of the glue stiffened polycarbonate film, i.e., $4(1+j \eta) \mathrm{GPa}$, where $\eta$ represents the loss factor. As indicated in Eq. (4), $\eta$ can significantly affect the acoustic impedance $Z^{\mathrm{A}}$ of EA-FET in resonant states. Here, EA-FET with the open circuit is adopted in simulations to investigate the effects introduced by different $\eta$ on the acoustic transmittance $\tau$. It can be found in Fig. 6(b) that the peak values of $\tau$ decrease with the increase of $\eta$. When $\eta$ is 0.02 , the simulation results are in good agreement with the experimental results. On the one hand, it indicates that the overall damping of our EA-FET sample is about 0.02 ; on the other hand, it again confirms the reliability of the finite-element model. To attain a great drain velocity under the open-circuit configuration, the damping of the entire system should be controlled as little as possible. 

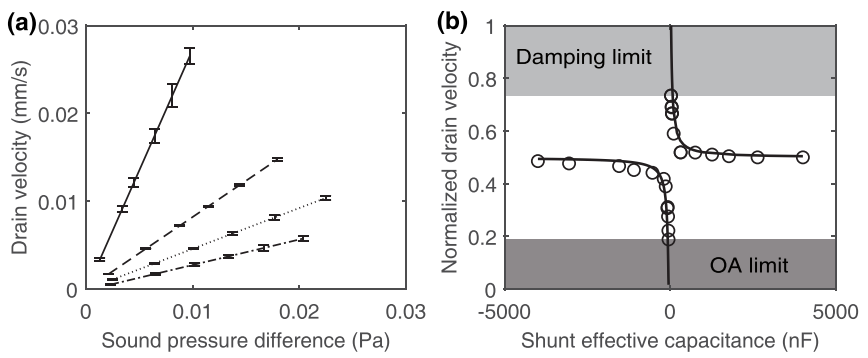

FIG. 7. Intrinsic output and transfer properties of the scaleddown EA-FET sample operating at $1240 \mathrm{~Hz}$. The experimental results in (a) correspond to four configurations, i.e., open circuit (solid line), shunt effective capacitance $C^{\mathrm{EL}}=-80 \mathrm{nF}$ (dashed line), $-55 \mathrm{nF}$ (dotted line), and $-48 \mathrm{nF}$ (dash-dotted line). Error bars correspond to the standard deviations resulting from the least-squares fit over three scaled-down EA-FET samples. Experimental and simulated results in (b) are represented by circles and curves, respectively. The drain velocities are normalized to the drain velocity in the case of total transmission.

\section{APPENDIX C: SCALED-DOWN SAMPLE AND ENHANCEMENT-TYPE EA-FET}

Since the proposed EA-FET operates in the linear regime, reducing the geometrical size in the structure would increase the operational frequency. Therefore, by increasing the operational frequencies, the acoustic wavelengths of the processed signals could be reduced, which might be one of the directions to miniaturize and integrate EA-FET. In the scaleddown EA-FET sample (84\% of the large-sized EA-FET sample in dimension), the thickness and radius of the polycarbonate film, the PZT-52 piezoelectric patch, and the stainless steel mass are 0.254 and $18.5 \mathrm{~mm}, 0.2$ and $12.5 \mathrm{~mm}$, and 1.4 and $5 \mathrm{~mm}$, respectively. The equivalent capacitance of the piezoelectric patch in this scaled-down EA-FET sample is $36 \mathrm{nF}$, which is close to the lower bound of the present shunt effective capacitance. Thus, the present external electric circuit is still valid for measuring the intrinsic output and transfer properties of the scaled-down EA-FET sample. Through numerical modal analysis, we can obtain that the first-order transverse resonant frequency, i.e., the operating frequency, of the scaled-down EA-FET sample with the open circuit is $1240 \mathrm{~Hz}$.

Figures 7(a) and 7(b) show the intrinsic output and transfer properties of the scaled-down EA-FET sample, respectively. We can find that the scaled-down EA-FET sample resembles the large-sized EA-FET sample in terms of both the intrinsic output and transfer properties. Specifically, the results shown in Fig. 7(a) confirm that the scaled-down EA-FET sample still has significant linear operating characteristics. The scaled-down EA-FET sample operating under the opencircuit configuration can achieve the maximum slope, i.e., the minimum acoustic impedance, and the resulting maximum drain velocity. In Fig. 7(b), the intrinsic transfer properties of the scaled-down EA-FET sample show a similar hyperbolic feature to that of the large-sized EA-FET sample. Both the damping limit and the OA limit still exist, and the numerical predictions agree well with the experimental results except for the two limits. These similarities not only show
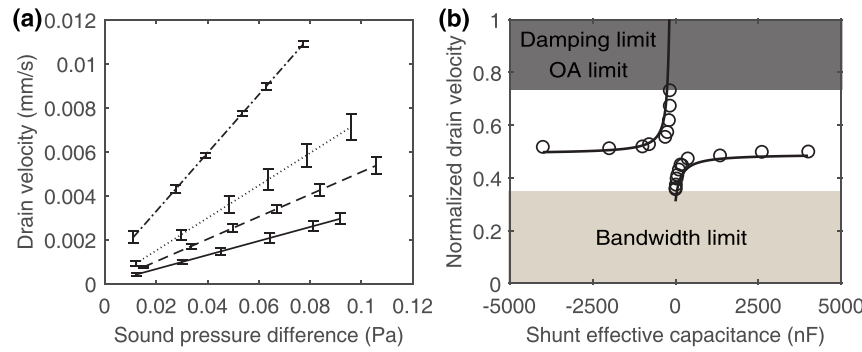

FIG. 8. The intrinsic output and transfer properties of the same EA-FET sample operating at $820 \mathrm{~Hz}$. The experimental results in (a) correspond to four configurations, i.e., open circuit (solid line), shunt effective capacitance $C^{\mathrm{EL}}=-200 \mathrm{nF}$ (dashed line), $-120 \mathrm{nF}$ (dotted line), and $-80 \mathrm{nF}$ (dash-dotted line). Error bars correspond to the standard deviations resulting from the least-squares fit over three EA-FET samples. Circles and curves in (b) represent the experimental and simulated results, respectively. The drain velocities are normalized to the drain velocity in the case of total transmission.

the universality of the operating characteristics of EA-FET, but they also demonstrate the scalability of the proposed EA-FET.

Due to the physics of wave-based devices, the EA-FET sample discussed in Figs. 2(b) and 2(c) can also be analogous to the enhancement-type MOSFET, in which there is not any channel preconstructed physically, and it requires an applied voltage to create a channel for conductance. It is immediately evident that the enhancement-type EA-FET is normally off, that is, when the shunt effective capacitance is made 0 (the open circuit), the enhancement type EA-FET does not conduct. Here, as shown in Fig. 8, the incident frequency is adjusted from 910 to $820 \mathrm{~Hz}$ to explore the intrinsic output and transfer properties of the same EA-FET sample.

The intrinsic output properties shown in Fig. 8(a) demonstrate that the enhancement-type EA-FET also operates in the linear regime. For each configuration of the external electric circuit, the drain velocity is proportional to the sound pressure difference. Compared with the depletion-type EA-FET, the drain velocity of the enhancement-type EA-FET is the lowest under the open-circuit configuration. In addition, as the shunt effective capacitance decreases, the slope of each line increases, indicating that the acoustic impedance of the enhancement type EA-FET decreases, thereby increasing the acoustic flux.

In contrast to the depletion-type EA-FET, the enhancement-type EA-FET exhibits very different intrinsic transfer properties as shown in Fig. 8(b). Although the intrinsic transfer properties of the enhancement-type EA-FET exhibit the hyperbolic feature, the orientations of the two branches corresponding to the shunted negative and positive capacitances show an inverse relationship with those of the depletion-type EA-FET, which results in the overlap of the damping limit and the OA limit. Note that a bandwidth limit exists for the enhancement-type EA-FET. The acoustic transmittance at $820 \mathrm{~Hz}$ of the enhancement-type EA-FET with the open circuit is responsible for the formation and level of the bandwidth limit. As shown in Fig. 6(b), due to the 
bandwidth influence of the peak around $910 \mathrm{~Hz}$, the acoustic transmittance is not ideally close to 0 at $820 \mathrm{~Hz}$, which implies that this EA-FET sample allows a certain sound to pass, resulting in the inability to achieve the complete acoustic cutoff. One possible solution is to design a sufficiently low acoustic transmittance at the initial operating frequency, for instance, taking the antiresonance frequency of EA-FET as the initial operating frequency.
[1] E. R. MacQuarrie, C. Simon, S. Simmons, and E. Maine, The emerging commercial landscape of quantum computing, Nat. Rev. Phys. 2, 596 (2020).

[2] F. Zangeneh-Nejad, D. L. Sounas, A. Alù, and R. Fleury, Analogue computing with metamaterials, Nat. Rev. Mater. 6, 207 (2021).

[3] H. Yasuda, P. R. Buskohl, A. Gillman, T. D. Murphey, S. Stepney, R. A. Vaia, and J. R. Raney, Mechanical computing, Nature (London) 598, 39 (2021).

[4] A. Babaee, A. Momeni, A. Abdolali, and R. Fleury, Parallel Analog Computing Based on a $2 \times 2$ Multiple-Input MultipleOutput Metasurface Processor with Asymmetric Response, Phys. Rev. Appl. 15, 044015 (2021).

[5] S. Alexandre, M. Francesco, C. Giuseppe, G. Vincenzo, A. Andrea, and E. Nader, Performing mathematical operations with metamaterials, Science 343, 160 (2014).

[6] S. Zuo, Q. Wei, Y. Tian, Y. Cheng, and X. Liu, Acoustic analog computing system based on labyrinthine metasurfaces, Sci. Rep. 8, 10103 (2018).

[7] G. Ptitcyn, M. S. Mirmoosa, and S. A. Tretyakov, Timemodulated meta-atoms, Phys. Rev. Research 1, 023014 (2019).

[8] S. Abdollahramezani, O. Hemmatyar, and A. Adibi, Metaoptics for spatial optical analog computing, Nanophotonics $\mathbf{9}$, 4075 (2020).

[9] Z. Lv, P. Liu, and Y. Pei, Temporal acoustic wave computational metamaterials, Appl. Phys. Lett. 117, 131902 (2020).

[10] S. R. Sklan, Splash, pop, sizzle: Information processing with phononic computing, AIP Adv. 5, 053302 (2015).

[11] M. Maldovan, Sound and heat revolutions in phononics, Nature (London) 503, 209 (2013).

[12] S. A. Cummer, J. Christensen, and A. Alù, Controlling sound with acoustic metamaterials, Nat. Rev. Mater. 1, 16001 (2016).

[13] H. Nassar, B. Yousefzadeh, R. Fleury, M. Ruzzene, A. Alù, C. Daraio, A. N. Norris, G. Huang, and M. R. Haberman, Nonreciprocity in acoustic and elastic materials, Nat. Rev. Mater. 5, 667 (2020).

[14] B. Liang, B. Yuan, and J. Cheng, Acoustic Diode: Rectification of Acoustic Energy Flux in One-Dimensional Systems, Phys. Rev. Lett. 103, 104301 (2009).

[15] B. Liang, X. S. Guo, J. Tu, D. Zhang, and J. C. Cheng, An acoustic rectifier, Nat. Mater. 9, 989 (2010).

[16] N. Boechler, G. Theocharis, and C. Daraio, Bifurcation-based acoustic switching and rectification, Nat. Mater. 10, 665 (2011).

[17] X.-F. Li, X. Ni, L. Feng, M.-H. Lu, C. He, and Y.-F. Chen, Tunable Unidirectional Sound Propagation through a SonicCrystal-Based Acoustic Diode, Phys. Rev. Lett. 106, 084301 (2011).

[18] A. S. Gliozzi, M. Miniaci, A. O. Krushynska, B. Morvan, M. Scalerandi, N. M. Pugno, and F. Bosia, Proof of concept of a frequency-preserving and time-invariant metamaterial-based nonlinear acoustic diode, Sci. Rep. 9, 9560 (2019).

[19] D. Hatanaka, I. Mahboob, K. Onomitsu, and H. Yamaguchi, A phonon transistor in an electromechanical resonator array, Appl. Phys. Lett. 102, 213102 (2013).

[20] S. R. Sklan and J. C. Grossman, Phonon diodes and transistors from magneto-acoustics, New J. Phys. 16, 053029 (2014).

[21] B. Liang, W. Kan, X. Zou, L. Yin, and J. Cheng, Acoustic transistor: Amplification and switch of sound by sound, Appl. Phys. Lett. 105, 083510 (2014).

[22] M. Malishava and R. Khomeriki, All-Phononic Digital Transistor on the Basis of Gap-Soliton Dynamics in an Anharmonic Oscillator Ladder, Phys. Rev. Lett. 115, 104301 (2015).

[23] J. Liang, B.-H. Liu, H.-X. Zhang, H. Zhang, M.-L. Zhang, D.-H. Zhang, and W. Pang, Monolithic acoustic graphene transistors based on lithium niobate thin film, J. Phys. Appl. Phys. 51, 204001 (2018).

[24] T. Zhang, Y. Cheng, J. Guo, J. Xu, and X. Liu, Acoustic logic gates and boolean operation based on selfcollimating acoustic beams, Appl. Phys. Lett. 106, 113503 (2015).

[25] S.-D. Zhao, A.-L. Chen, Y.-S. Wang, and C. Zhang, Continuously Tunable Acoustic Metasurface for Transmitted Wavefront Modulation, Phys. Rev. Appl. 10, 054066 (2018).

[26] X. Chen, P. Liu, Z. Hou, and Y. Pei, Magnetic-control multifunctional acoustic metasurface for reflected wave manipulation at deep subwavelength scale, Sci. Rep. 7, 9050 (2017).

[27] S. Liang, T. Liu, H. Gao, Z. Gu, S. An, and J. Zhu, Acoustic metasurface by layered concentric structures, Phys. Rev. Research 2, 043362 (2020).

[28] W. K. Cao, C. Zhang, L. T. Wu, K. Q. Guo, J. C. Ke, T. J. Cui, and Q. Cheng, Tunable Acoustic Metasurface for ThreeDimensional Wave Manipulations, Phys. Rev. Appl. 15, 024026 (2021).

[29] X.-D. Fan and L. Zhang, Acoustic orbital angular momentum Hall effect and realization using a metasurface, Phys. Rev. Research 3, 013251 (2021).

[30] S. Alagoz and B. Baykant Alagoz, Sonic crystal acoustic switch device, J. Acoust. Soc. Am. 133, EL485 (2013).

[31] F. Li, P. Anzel, J. Yang, P. G. Kevrekidis, and C. Daraio, Granular acoustic switches and logic elements, Nat. Commun. 5, 5311 (2014).

[32] S. Babaee, N. Viard, P. Wang, N. X. Fang, and K. Bertoldi, Harnessing deformation to switch on and off the propagation of sound, Adv. Mater. 28, 1631 (2016).

[33] O. R. Bilal, A. Foehr, and C. Daraio, Bistable metamaterial for switching and cascading elastic vibrations, Proc. Natl. Acad. Sci. USA 114, 4603 (2017).

[34] T. Devaux, A. Cebrecos, O. Richoux, V. Pagneux, and V. Tournat, Acoustic radiation pressure for nonreciprocal transmission and switch effects, Nat. Commun. 10, 3292 (2019). 
[35] Y. Zhang, C. Wang, and L. Huang, A tunable electromagnetic acoustic switch, Appl. Phys. Lett. 116, 183502 (2020).

[36] P. Cao, Y. Zhang, S. Zhang, W. Ou, S. G. Mosanenzadeh, and N. X. Fang, Switching Acoustic Propagation via Underwater Metasurface, Phys. Rev. Appl. 13, 044019 (2020).

[37] X. Jiang, B. Liang, J.-C. Cheng, and C.-W. Qiu, Twisted acoustics: Metasurface-enabled multiplexing and demultiplexing, Adv. Mater. 30, 1800257 (2018).

[38] F. Liu, W. Li, Z. Pu, and M. Ke, Acoustic waves splitter employing orbital angular momentum, Appl. Phys. Lett. 114, 193501 (2019).

[39] H. Ni, X. Fang, Z. Hou, Y. Li, and B. Assouar, High-efficiency anomalous splitter by acoustic meta-grating, Phys. Rev. B 100, 104104 (2019).

[40] T. Liu, G. Ma, S. Liang, H. Gao, Z. Gu, S. An, and J. Zhu, Single-sided acoustic beam splitting based on parity-time symmetry, Phys. Rev. B 102, 014306 (2020).

[41] T. Gu, Y. Cheng, Z. Wen, E. H. Ei Boudouti, Y. Jin, Y. Li, and B. Djafari-Rouhani, Induced transparency based subwavelength acoustic demultiplexers, J. Phys. Appl. Phys. 54, 175301 (2021).

[42] B. S. Beck, K. A. Cunefare, M. Collet, and M. Ruzzene, Active vibration control of a stiffened panel through application of negative capacitance shunts, Proc. SPIE 7977, 79770C (2011).

[43] X. Wang, J. Xu, J. Ding, C. Zhao, and Z. Huang, A compact and low-frequency acoustic energy harvester using layered acoustic metamaterials, Smart Mater. Struct. 28, 025035 (2019).

[44] P. Celli and S. Gonella, Tunable directivity in metamaterials with reconfigurable cell symmetry, Appl. Phys. Lett. 106, 091905 (2015).
[45] B.-I. Popa and S. A. Cummer, Non-reciprocal and highly nonlinear active acoustic metamaterials, Nat. Commun. 5, 3398 (2014).

[46] R. Zhu, Y. Y. Chen, M. V. Barnhart, G. K. Hu, C. T. Sun, and G. L. Huang, Experimental study of an adaptive elastic metamaterial controlled by electric circuits, Appl. Phys. Lett. 108, 011905 (2016).

[47] K. Yi and M. Collet, Broadening low-frequency bandgaps in locally resonant piezoelectric metamaterials by negative capacitance, J. Sound Vib. 493, 115837 (2021).

[48] G. Ma, M. Yang, S. Xiao, Z. Yang, and P. Sheng, Acoustic metasurface with hybrid resonances, Nat. Mater. 13, 873 (2014).

[49] M. Date, M. Kutani, and S. Sakai, Electrically controlled elasticity utilizing piezoelectric coupling, J. Appl. Phys. 87, 863 (2000).

[50] N. W. Hagood and A. von Flotow, Damping of structural vibrations with piezoelectric materials and passive electrical networks, J. Sound Vib. 146, 243 (1991).

[51] B. H. Song and J. S. Bolton, A transfer-matrix approach for estimating the characteristic impedance and wave numbers of limp and rigid porous materials, J. Acoust. Soc. Am. 107, 1131 (2000).

[52] D. M. Pozar, Microwave Engineering, 4th ed. (John Wiley \& Sons, New York, 2011), p. 192.

[53] A. A. Maznev, A. G. Every, and O. B. Wright, Reciprocity in reflection and transmission: What is a 'phonon diode'?, Wave Motion 50, 776 (2013).

[54] See Supplemental Material at http://link.aps.org/supplemental/ 10.1103/PhysRevResearch.3.043206 for the video of the realtime acoustic binary encoding demonstration. 